\title{
Effect of strength and proprioception training on eversion to inversion strength ratios in subjects with unilateral functional ankle instability
}

\author{
T W Kaminski, B D Buckley, M E Powers, T J Hubbard, C Ortiz
}

See end of article for authors' affiliations

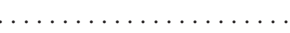

Correspondence to: Dr Kaminski, Department of Health, Nutrition \&

Exercise Science, Human Performance Laboratory, University of Delaware, 541 South College

Avenue, Newark, DE

19716, USA

kaminski@udel.edu

Accepted 25 September 2002

\begin{abstract}
Objectives: To examine the effect of six weeks of strength and proprioception training on eversion to inversion isokinetic strength ratios ( $\mathrm{E} / \mathrm{I}$ ratios) in subjects with unilateral functional ankle instability.

Methods: Thirty eight subjects were randomly assigned to one of four treatment groups: strength training $(\mathrm{S})$; proprioception training $(\mathrm{P})$; strength + proprioception training $(\mathrm{B})$; control $(\mathrm{C})$. Isokinetic strength was tested before and after training using a Kin Com 125 automatic positioning isokinetic dynamometer. Subtalar joint eversion and inversion motions were tested both concentrically and eccentrically through a range of motion involving $40^{\circ}$. All peak torque and average torque values were normalised for body mass. E/I ratios were calculated from average torque and peak torque measures by taking the concentric eversion value and combining it with the eccentric inversion value. Data were analysed using a mixed model analysis of variance with repeated measures on the test factor. Average torque and peak torque $\mathrm{E} / \mathrm{I}$ ratios at 30 and $120 \%$ s were analysed separately.

Results: There were no significant differences in average torque and peak torque $E / I$ ratios of the functionally unstable ankle for any of the groups after training compared with before.

Conclusions: Six weeks of strength and proprioception training (either alone or combined) had no effect on isokinetic measures of strength in subjects with self reported unilateral functional instability. Further studies examining this agonist (concentric) to antagonist (eccentric) muscle group strength ratio are needed.
\end{abstract}

$\mathrm{T}$ he high incidence of ankle sprain in athletes means that much time and energy are spent on treatment and rehabilitation. In cutting and jumping sports such as volleyball, football, soccer, and basketball, lateral ankle sprains are one of the most common injuries. ${ }^{12}$ Even more alarming is that after an initial ankle sprain, the athlete is more susceptible to long term disability and degeneration. ${ }^{3-6}$

Freeman ${ }^{7}$ first coined the term "functional ankle instability" (FAI) indicating that it was the subjective feeling of "giving way" after repeated episodes of ankle sprain. Tropp ${ }^{8}$ later refined the term, indicating that it was joint motion beyond voluntary control, but not exceeding physiological range of motion. The pathogenesis of FAI is thought to involve mechanical, muscular, and sensorimotor deficiencies. ${ }^{9}$ Bosein and colleagues ${ }^{10}$ were the first to report that peroneal muscle weakness was the most significant factor contributing to recurrent ankle sprains. Since then there has been a plethora of reports examining strength deficits in those with FAI, with some supporting muscle weakness as a cause $\mathrm{e}^{11-13}$ and others refuting this. ${ }^{14-16}$ Despite contradictory findings, peroneal strengthening has been advocated for many years in the rehabilitation of both acute and chronic ankle sprains. ${ }^{10}$

Co-activation of antagonistic muscle groups has been identified as an important factor influencing dynamic joint stability. ${ }^{17}{ }^{18}$ Traditionally, the strength ratios between these muscle groups have received considerable attention from clinicians during preseason examinations and in monitoring rehabilitation progress. With the advent of active isokinetic dynamometers, clinicians are now able to assess both concentric and eccentric muscle actions, which enables examination of the reciprocal muscle group ratios. In other words, these isokinetic dynamometers allow the examination of the co-activation of agonist and antagonist muscle groups at a specific joint. Hole et $a l^{19}$ and Aagaard et $a l^{20}$ reported the use of agonist to antagonist muscle group ratios for the quadriceps and hamstring muscles. Aagaard $e a^{20}$ argue that providing strength information on the nature of the agonist and antagonist muscle group relation will create a more complete/ clearer picture of the true dynamic nature of the muscles' function. However, few studies have examined reciprocal muscle group ratios in the ankle joint, ${ }^{13162122}$ and fewer yet have examined the "dynamic strength control" ratios incorporating both concentric and eccentric muscle actions. ${ }^{1923-25}$ Interestingly, muscle group ratios in the shoulder (abductor/ adductor and external rotator/internal rotator) and knee (hamstring/quadriceps) have been used for many years to evaluate rehabilitation progress. It was only recently that we began to express similar muscle group ratios in the ankle. Perhaps in the near future, ankle joint strength ratios will be used, much like those in both the shoulder and knee joints, to detect muscle imbalances in an attempt to prevent injury.

Although the effects of proprioception training (coordination, ankle disk training, postural control) in those with and without FAI have been investigated, ${ }^{26}{ }^{27}$ few studies have examined the effects of strength training alone ${ }^{28} 29$ or combined with proprioception training..$^{30}$ These studies examined a variety of both strength and proprioception measurements including static and dynamic balance (sway), along with isometric and isokinetic strength. In the study of Blackburn et al, ${ }^{30}$ subjects were assigned to one of four treatment groups: control, strength training, proprioception training, and combined strength and proprioception training. The effects of training on measures of balance and not strength were investigated. Conversely, Docherty et $a l^{29}$ examined the effects of six weeks of ankle strength training on measures of joint position sense and strength development.

Abbreviations: FAl, functional ankle instability; E/I ratio, eversion to inversion isokinetic strength ratio 


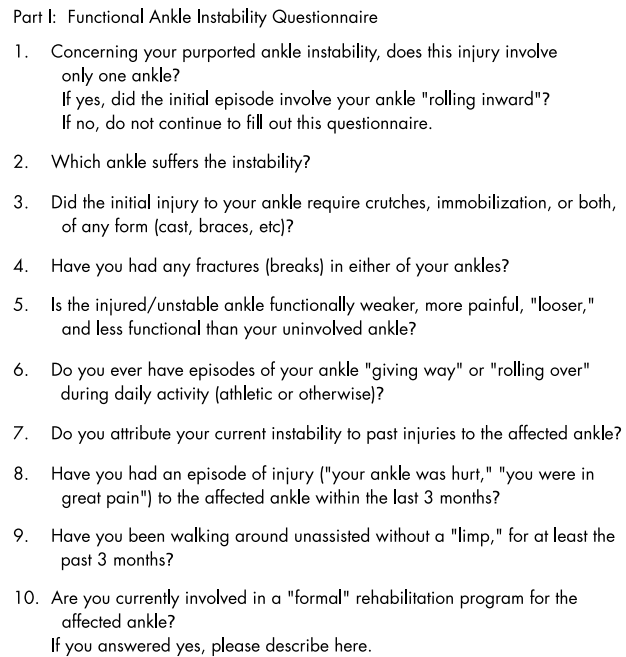

11. Can you describe a symptom(s) of your ankle "giving way"?

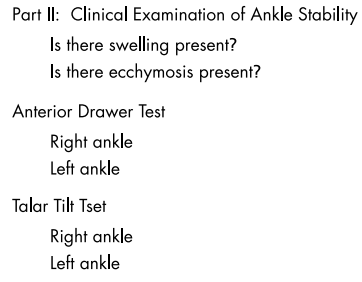

Figure 1 Questionnaire to determine if subjects met the criteria for functional ankle instability (FAI). Reproduced with permission from Hubbard TJ, Kaminski TW. Kinesthesia is not offected by functional ankle instability status. Journal of Athletic Training 2002;37:481-6.

Therefore, using a pretest-post-test randomised group design, we examined the effect of six weeks of strength and proprioception training on eversion to inversion isokinetic strength ratios (E/I ratios) in subjects with unilateral FAI. We hypothesised that subjects participating in a combined programme of strength and proprioception training would show greater improvement in E/I ratios than any of the other groups.

\section{METHODS \\ Subjects}

Thirty eight subjects (22 men, 16 women; mean (SD) age 21.6 (2.9) years, height $174.1(9.8) \mathrm{cm}$, weight 78.2 (16.6) kg) who met our stringent FAI criteria agreed to participate in the study. They were physically active and able to maintain their activity level regardless of their FAI status. Before participation, they signed an informed consent agreement that had been approved by the university's institutional review board. All subjects were examined by the same clinician (BB) and found to have no mechanical instability before participating. To qualify as having FAI, the subject had to meet the criteria outlined in our questionnaire (fig l).

Subjects who qualified for participation were randomly assigned to one of four treatment groups: strength training (S); proprioception training $(\mathrm{P})$; strength + proprioception training (B); control (C). The S group participated in six weeks of plantar flexion, dorsiflexion, inversion, and eversion strength training using Thera-Band (Hygenic Corporation,

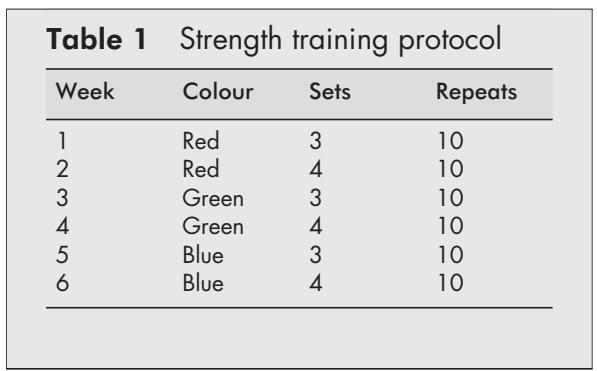

Ohio, USA) elasticated bands. The P group participated in six weeks of proprioception training which involved "T-band kicks". ${ }^{31}$ The B group performed a combination of both training protocols, and the $\mathrm{C}$ group refrained from any formal ankle training.

\section{Strength and proprioception training protocols}

The strength and proprioception training protocols were based on common clinical practice and experiences. Each protocol involved the application of ordinary strength and proprioception rehabilitation techniques. All three training groups exercised three times a week for six weeks. The time spent in each session varied according to the group. All subjects trained using their affected ankle only. Each training session was supervised by one of the members of the research team.

The S group trained using a progressive resistance protocol using Thera-Band elasticated bands. Subjects progressed weekly in sets and/or resistance throughout the training period (table 1). The strength training session was performed with the subject seated on the floor with the knee extended. The Thera-Bands were doubled and attached to a hook on the wall. The looped end was secured to the foot while the subject performed the plantar flexion, dorsiflexion, inversion, and eversion motions. Subjects were instructed to concentrate on performing only the movements at the ankle joint and not add any extraneous movements from either the knee or hip joints. The training resistance was determined by calculating $70 \%$ of the resting length of the doubled Thera-Band. This distance was then added to the resting length of the band, and a mark was placed on the floor to which the Thera-Band had to be stretched for the subject to perform the exercise routine (fig 2 ). Regardless of the colour (resistance) of the band, all strength training exercises were performed with the bands elongated to $70 \%$. This protocol was designed to ensure that all subjects trained with a consistent and progressive amount of resistive force. All subjects progressed uniformly throughout the six week training period. Using a set/repetition protocol similar to that described by Docherty et al, ${ }^{29}$ each subject performed 10 repetitions per set.

The $\mathrm{P}$ group trained using a progressive protocol with Thera-Bands. A T-band kicks protocol modified from that originally described by Tomaszewski ${ }^{31}$ was used. The TheraBands were attached to a hook on the wall but were not double looped. The subject placed their uninvolved foot through the loop in the Thera-Band opposite the loop attached to the wall. The subject then stood barefoot while balancing on the FAI ankle (fig 3). The uninvolved foot was used to perform the kicking motions only. The kicking motions consisted of short and quick oscillations. Consistency of oscillation was controlled by having the subject perform the T-band kicks to the beat of a metronome set at 80 beats/minute. The subject was asked to perform two sets of 25 T-band kicks in four different directions of hip motion (flexion, extension, abduction, and adduction) while balancing on the FAI ankle. If the subject was able to complete 2 sets $\times 25$ repetitions in each direction without losing balance or touching the uninvolved foot to the ground, they would change Thera-Band colour (resistance) at their next training session. All subjects started using the TheraBand colour red. Once a subject reached the highest resistance 

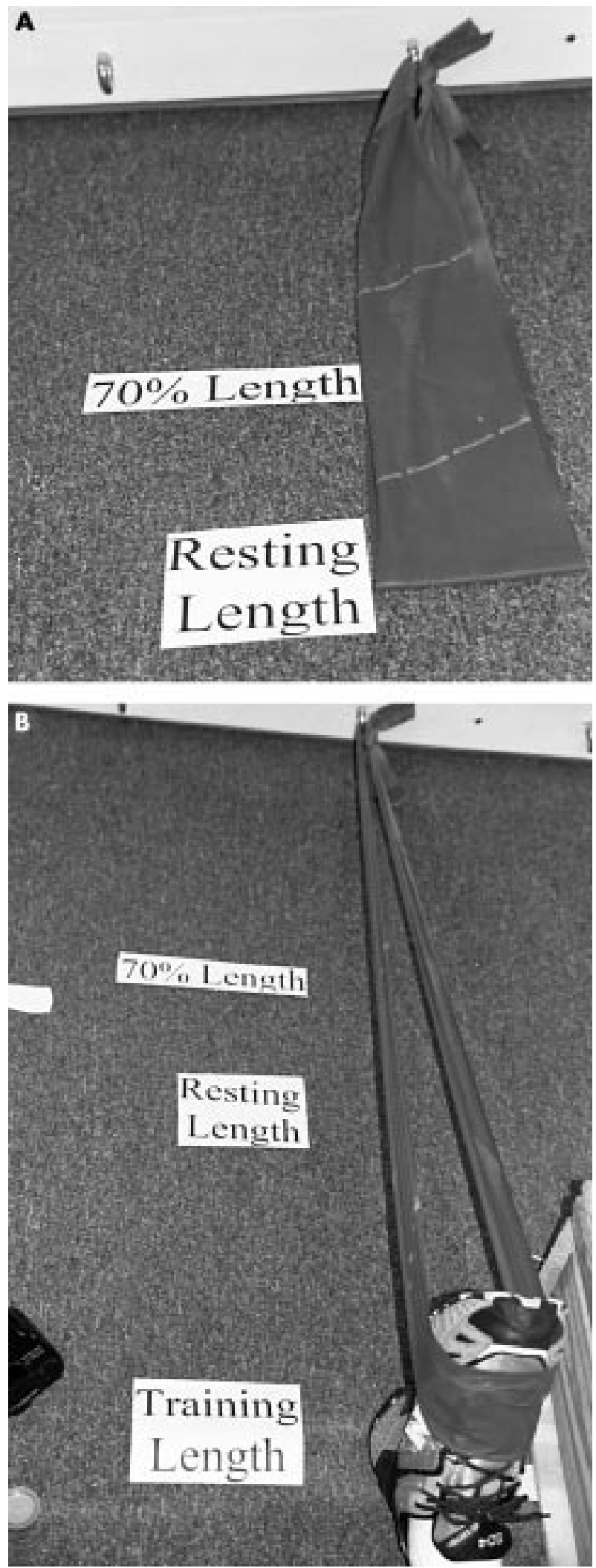

Figure 2 (A) 70\% of the Thera-Band resting length was determined to calculate the training distance. (B) Training with the band elongated $70 \%$.

(gold), repetitions were increased by 10 if progression was necessary.

Subjects in the $\mathrm{B}$ group performed the above exercise protocols in conjunction with one another. As expected this

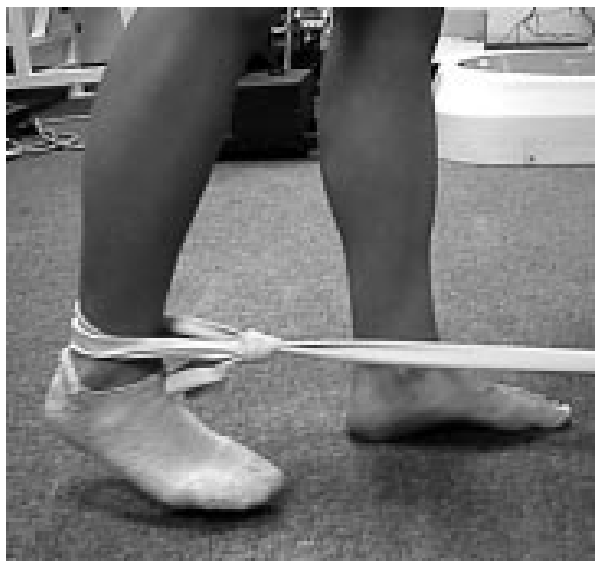

Figure 3 "T-band kicks" training.

training session required more time to complete the entire routine. The order of training (strength or proprioception) was randomised at each session. Subjects in the C group did not participate in any training.

\section{Strength assessment}

Isokinetic strength was assessed before and after the six week training period. The Kin Com 125 automatic positioning isokinetic dynamometer (Chattanooga Group, Hixson, Tennessee, USA) was used to assess peak and average torque for the motions of subtalar joint eversion and inversion. Kin Com dynamometers allow precise and reliable measurement and storage of data from isokinetic, isotonic, and isometric muscular actions. ${ }^{32}{ }^{33}$ In an earlier study, we examined the reliability of isokinetic strength measurements from the Kin Com and calculated intraclass correlation coefficients ranging from 0.69 to $0.91 .{ }^{33}$ The ankle motions were tested with the subject seated on the dynamometer chair. All tests were performed with the subjects wearing shoes. The subjects were stabilised in the chair according to the manufacturer's guidelines, with straps securing the chest and waist. The isokinetic dynamometer was moved to the appropriate position and height for ankle strength testing using the automatic positioning function of the dynamometer. The universal stabiliser was used to position and hold the lower leg and to help to prevent any unwanted muscle substitution. The foot was securely fastened into the ankle inversion/eversion footplate attachment using Velcro closures. The start and stop angles for eversion to inversion motion were set at $15^{\circ}$ and $25^{\circ}$ respectively, and the inversion to eversion angles were set at $25^{\circ}$ and $15^{\circ}$ respectively. We chose these range of motion stops so that the test protocol was standardised for all subjects. More importantly, when the overlay procedure on the Kin Com dynamometer was used, these stops enabled the subject to generate enough force to start the dynamometer moving during the eccentric phase of the movement. Often, when subjects are at the extreme of their inversion or eversion range, they are unable to generate enough force to initiate movement of the dynamometer lever arm in the opposite direction (eccentric phase), even when the force limits are set to a minimum. This procedure allowed all subjects to comfortably perform the required motion within a $40^{\circ}$ range.

Isokinetic tests were performed at 30 and $120 \%$ on both ankles; however, only data from the involved ankle were used in the statistical analyses. To become familiar with the isokinetic exercise concept, especially the eccentric mode, each subject was allowed three submaximal (50\% capacity) warm up repetitions at each velocity. A two minute rest was provided at the end of the practice session. The order of test velocity $(30 \%$ or $120 \%$ s) and ankle motion (eversion or inversion) was randomised using a flip of the coin to minimise any potential 
learning effects. Three maximal eccentric and concentric test repetitions were completed through the $40^{\circ}$ range of motion at both test velocities. With the overlay (interrupted) sequence protocol of the Kin Com software package, each of these repetitions was completed individually with a 15 second pause between repetitions. Subjects were instructed to provide maximal effort throughout the entire repetition and were given both verbal and visual feedback and encouragement. Repetitions were repeated if the torque curve did not closely match the previous attempt. This was to ensure that the subject was exerting maximal effort with each test repetition. A one minute rest period was given between tests of each motion, velocity, and side. At the conclusion of testing, peak and average torque data were extracted from the torque curves and used in the conversion to E/I ratios. The E/I ratios were then derived by taking the concentric eversion value and dividing it by the eccentric inversion value for either peak torque or average torque on each ankle (FAI $v$ uninjured). This ratio expresses the viewpoint of the evertors acting concentrically to counteract the violent inversion mechanism in an open kinetic chain, and/or the invertors acting eccentrically to slow the lateral displacement of the tibia in a closed kinetic chain. These E/I ratios were then subjected to statistical analysis.

\section{Statistical analysis}

The independent variables included test (before and after) and group status ( S, P, B, and C). The four dependent variables included peak torque E/I ratio data at 30 and $120^{\circ}$ s and average torque E/I ratio data at 30 and $120 \%$ from the involved ankles. A $2 \times 4$ mixed model analysis of variance with repeated measures on the test factor was used to determine if differences in involved (FAI) ankles existed between measurements before and after training for peak torque and average torque E/I ratios. Separate analyses were conducted on each of the four dependent variables. The between subject factor was group status, and the within subject factor included test. SPSS 9.0.0 for Windows (SPSS, Chicago, Illinois, USA) was used for statistical analysis. An $\alpha$ level of $p<0.05$ was set a priori for all analyses.

\section{RESULTS}

Average torque E/I ratios ranged from 0.46 to 2.54 , and peak torque E/I ratios from 0.35 to 2.38. Table 2 gives E/I ratios for both average torque and peak torque before and after training.

Analysis of variance for the average torque E/I ratio (30 and $120^{\circ} / \mathrm{s}$ ) did not detect any significant differences after the training for any of the four groups. The test by group $F$ statistic for the ratios at $30^{\circ} / \mathrm{s}$ was $F_{(3,34)}=2.173, \mathrm{p}=0.109$; at $120^{\circ} / \mathrm{s}$, $F_{(3,34)}=1.237, \mathrm{p}=0.311$. In addition, there were no significant main effects for either of the group factors at $30^{\circ} / \mathrm{s}\left(F_{(3,34)}=\right.$
$0.302, \mathrm{p}=0.824)$ and $120^{\circ} / \mathrm{s}\left(F_{(3,34)}=1.475, \mathrm{p}=0.239\right)$ or test factors at $30 \% \mathrm{~s}\left(F_{(1.34)}=0.572, \mathrm{p}=0.455\right)$ and $120^{\circ} / \mathrm{s}\left(F_{(1,34)}=\right.$ $3.063, \mathrm{p}=0.089$ ).

Analysis of variance for peak torque E/I ratio (30 and $120 \%$ ) did not detect any significant differences after training for any of the four groups. The test by group F statistic for the ratios at $30^{\circ} / \mathrm{s}$ was $F_{(3,34)}=1.544, \mathrm{p}=0.221 ;$ at $120^{\circ} / \mathrm{s}, F_{(3,34)}=2.123, \mathrm{p}=$ 0.116 . In addition, there were no significant main effects for either of the group factors at $30^{\circ} / \mathrm{s}\left(F_{(3,34)}=0.098, \mathrm{p}=0.961\right)$ and $120^{\circ} / \mathrm{s}\left(F_{(3,34)}=1.434, \mathrm{p}=0.250\right)$ or test factors at $30 \% \mathrm{~s}$ $\left(F_{(1,34)}=0.008, \mathrm{p}=0.929\right)$ and $120^{\circ} / \mathrm{s}\left(F_{(1,34)}=3.003, \mathrm{p}=\right.$ 0.092).

A post hoc power and effect size analysis was performed after the indication of "non-significant" findings. This is best illustrated when the effect sizes for the differences between the values before and after training at 30 and $120 \%$ s were compared for each of the four groups (table 2). The effect sizes ranged from 0.07 to 0.59 for average torque and 0.12 to 0.62 for peak torque. Interestingly, half of the effect size values in both data sets fell below 0.30 (low levels), and the other half fell between 0.35 and 0.65 (moderate levels). The highest effect sizes were found when comparing the E/I ratios before and after training in the B group. It should be borne in mind that the smaller the effect size, the smaller the magnitude of difference between the conditions. Using these effect sizes, we performed a post hoc power calculation based on 10 subjects per group and recognised a power of 0.16 for both the average and peak torque data sets.

\section{DISCUSSION}

Contrary to what we had hypothesised, there were no significant differences in average torque and peak torque strength ratios in any of the groups. It appears that six weeks of controlled and supervised strength and proprioception training does not significantly affect measures of strength in a group of subjects with self reported unilateral FAI. Perhaps the training regimens used varied so much from the way in which strength was isokinetically measured that, even if differences did exist, the method of testing may not have been specific enough to detect them.

The lack of improvement in isokinetic strength, especially in the S group, may be best understood from a mode specificity standpoint. All subjects were tested for eversion and inversion strength while seated on an isokinetic dynamometer, yet the strength training protocol required our subjects to be seated on the floor and involved isotonic strength training exercises. These differences in both training and testing position, as well as type of strength training, may have led to the lack of significant changes in the E/I ratios in the S group after the six week training period. The issue of mode specificity is

Table $2 \mathrm{E} / \mathrm{I}$ ratios for average torque and peak torque before and after training on the functionally unstable ankle at two different velocities

\begin{tabular}{|c|c|c|c|c|}
\hline \multirow[b]{2}{*}{ Group } & \multicolumn{2}{|l|}{$30 \% / \mathrm{s}$} & \multicolumn{2}{|l|}{$120 \% \mathrm{~s}$} \\
\hline & Before & After & Before & After \\
\hline \multicolumn{5}{|l|}{ Average torque } \\
\hline Strength & $0.95(0.39)$ & $1.00(0.41)$ & $0.69(0.16)$ & $0.70(0.12)$ \\
\hline Proprioception & $1.17(0.58)$ & $0.96(0.33)$ & $0.82(0.28)$ & $0.80(0.27)$ \\
\hline Strength + proprioception & $0.99(0.22)$ & $1.07(0.21)$ & $0.74(0.15)$ & $0.84(0.19)$ \\
\hline Control & $1.14(0.32)$ & $1.08(0.27)$ & $0.82(0.23)$ & $0.92(0.25)$ \\
\hline \multicolumn{5}{|l|}{ Peak torque } \\
\hline Strength & $0.94(0.39)$ & $1.07(0.44)$ & $0.61(0.17)$ & $0.63(0.13)$ \\
\hline Proprioception & $1.10(0.59)$ & $0.92(0.28)$ & $0.75(0.20)$ & $0.70(0.21)$ \\
\hline Strength + proprioception & $0.99(0.24)$ & $1.06(0.30)$ & $0.66(0.14)$ & $0.75(0.15)$ \\
\hline Control & $1.10(0.39)$ & $1.06(0.29)$ & $0.71(0.17)$ & $0.81(0.20)$ \\
\hline
\end{tabular}

Values are mean (SD).

$E / I$ ratio, eversion to inversion isokinetic strength ratio. 
documented in the literature. ${ }^{34} 35$ To prove this point further, in contrast with our study, a recent study by Uh et al, ${ }^{28}$ showed significant improvements in isokinetic peak torque measurements in healthy ankles strength trained for eight weeks using a Cybex dynamometer. That study can be used to confirm the effectiveness of strength training on the same device used for strength test measurements before and after the training. Perhaps we would have detected similar changes if our subjects had trained on the Kin Com dynamometer.

Furthermore, we theorise that the Thera-Band strength training regimen used in our study was not rigorous enough and may not have provided an intense enough resistive stimulus to bring about significant improvements in the isokinetic $\mathrm{E} / \mathrm{I}$ ratios. One of the greatest difficulties that we encountered in the development of the Thera-Band strength and proprioception training protocols was the lack of objective evidence that supports their use in previous studies. Baker et al ${ }^{36}$ examined the effects of a six week T-band kicks training protocol on postural sway. Their protocol consisted of performing the kicks with the eyes open and progressing to eyes closed, with repetitions ranging from 30 to 50 . They concluded that the resistive tubing kicks did not significantly improve postural sway and suggested that a greater intensity of training may be necessary. We would also add that, because we only trained our subjects three times a week for a total of six weeks, the protocol itself may not have been strenuous enough to bring about a strength change adaptation, especially in a physically active population with FAI.

Interestingly, Docherty and colleagues ${ }^{29}$ reported improvements in eversion and dorsiflexion strength after six weeks of progressive resistance strength training using a protocol similar to ours. They used 20 subjects with a history of unilateral FAI. Their subjects also showed improvements in joint position sense measures, a finding the researchers attributed to enhancements in muscle spindle activity. One criticism we have of this report is that the inclusion criteria for functional instability were rather vague, especially with regard to the term "giving way". Moreover, they used a hand held dynamometer to test isometric strength, whereas we used an isokinetic dynamometer. Although their methods were valid and reliable, a strength measurement at one point in the range of motion does not provide as accurate an assessment of strength as measurement of strength throughout a defined range of motion.

In sharp contrast with the findings of Docherty et al, ${ }^{29}$ Holme and associates ${ }^{37}$ suggested that, six weeks after an ankle injury, subjects displayed a side to side deficit in isometric ankle strength and postural control. Several differences between the studies should be noted. Firstly all 92 subjects in this study had suffered acute lateral ankle sprains and had no history of FAI. Perhaps had they used subjects with FAI, the results after six weeks of training would have been similar to ours. They speculate that pain and inhibition may have played a role during the strength measurements at an early stage after injury. They were not able to pinpoint whether the decline in strength performance was attributable to a reduction in neural activity or muscle atrophy associated with disuse. In addition, the standardised rehabilitation programme consisted of a different set of exercises including balancing on both legs, figure of eight running, standing on a balance board while catching a ball, and standing on the inside and outside of their feet with eyes open and closed. This exercise routine is quite different from the resistance band routine used in our study. They too used isometric measurements of strength instead of isokinetic dynamometry, again assessing strength at only one point in the ankle range of motion.

On close examination of our power analysis, the results are not surprising especially as we had small $F$ values and effect sizes, with high p values. It would be useful to have a better understanding of what a "clinically significant" difference
Take home message

Six week strength and proprioception training protocols had no significant effect on isokinetic $E / l$ strength ratios. Clinicians need to consider intensity and duration when implementing such protocols, especially in the rehabilitation of unilateral FAI. More studies are needed to examine the usefulness of expressing $E / I$ ratios using concentric and eccentric strength data.

between strength ratios is. The differences used to determine these effect sizes are our actual, observed differences, yet until more research is performed using these ratios, a "clinically significant" difference from which to make comparisons and the appropriate effect size calculation will remain elusive.

Previous studies ${ }^{38} 39$ have shown improvements in postural stability after six to eight week periods of ankle proprioception training (ankle disk and balance activities), but our study was unable to show improvements in strength as a crossover effect from proprioception training either alone or combined with strength training. Sheth $\mathrm{et} \mathrm{al}^{40}$ showed that ankle disk training influenced reaction times of selected muscles during a simulated ankle sprain. However, the subjects were uninjured and free from any functional instability. Further study is needed to determine if such changes would be evident in a population with FAI.

\section{Conclusions}

As the use of elasticated bands in the rehabilitation of ankle sprains becomes more widespread, we are optimistic that there will be more objective evidence available to support their implementation, especially in strength training studies of the ankle. Researchers have examined the Thera-Band resistive tubing and its material properties in order to quantify the amount of force that is applied with a given colour and length. ${ }^{41}{ }^{42}$ This will allow therapists and clinicians to objectively develop a strength and proprioception training protocol. Further research is needed to examine the intensity of training and offer guidelines on how strenuous the training exercises must be to bring about changes in strength.

Equally important from a research perspective is the concept of mode specificity. Researchers examining the area of FAI need to recognise that when subjects are trained using a specific protocol, a strength testing protocol needs to be developed that closely matches that protocol. Special considerations should be given to positioning and mode of exercise.

\section{Authors' affiliations}

T W Kaminski , Sports Medicine and Athletic Training Department, Southwest Missouri State University, Springfield, Missouri, USA

B D Buckley, University of North Florida, Jacksonville, Florida, USA M E Powers, University of Florida, Gainesville, Florida, USA

T J Hubbard, Penn State University, State College, Pennsylvania, USA

C Ortiz, Gainesville Health and Fitness Center, Gainesville, Florida, USA

\section{REFERENCES}

1 Barker HB, Beynnon BD, Renstrom AFH. Ankle injury risk factors in sports. Sport Med 1997;23:69-74.

2 Garrick JG, Requa RK. The epidemiology of foot and ankle injuries in sports. Clin Podiatr Med Surg 1989;6:629-37.

3 Kannus $\mathbf{P}$, Renstrom P. Current concepts review: treatment for acute tears of the lateral ligaments of the ankle. J Bone Joint Surg $[\mathrm{Am}]$ $1991 ; 73: 305-12$

4 Smith RW, Reischl SF. Treatment of ankle sprains in young athletes. Am J Sports Med 1986;14:465-71.

5 Yeung MS, Chan K, So $\mathrm{CH}$, et al. An epidemiological survey on ankle sprain. Br J Sports Med 1994;28:1 12-16

6 Hertel J. Functional instability following lateral ankle sprain. Sports Med 2000;29:361-71.

7 Freeman MAR. Instability of the foot after injuries to the lateral ligaments of the ankle. J Bone Joint Surg $[\mathrm{Br}]$ 1965;47:678-85. 
8 Tropp H. Functional instability of the ankle joint [dissertation]. Linkoping (Sweden): Linkoping University, 1985.

9 Konradsen L, Magnusson P. Increased inversion angle replication error in functional ankle instability. Knee Surg Sports Traumatol Arthrosc 2000:8:246-51.

10 Bosien WR, Staples OS, Russell SW. Residual disability following acute ankle sprains. J Bone Joint Surg [Am] 1955;37:1237-43.

11 Staples OS. Result study of ruptures of lateral ligaments of the ankle. Clin Orthop 1972;85:50-8

12 Tropp H. Pronator weakness in functional instability of the ankle joint. Int J Sports Med 1986;7:291-4.

13 Wilkerson GB, Pinerola JJ, Caturano RW. Invertor vs evertor peak torque and power deficiencies associated with lateral ankle ligament injury. J Orthop Sports Phys Ther 1997;26:78-86.

14 Lentell GL, Bass B, Lopez D, et al. The contributions of proprioceptive deficits, muscle function, and anatomic laxity to functional instability of the ankle. J Orthop Sports Phys Ther 1995;21:206-15.

15 Kaminski TW, Perrin DH, Gansneder BM. Eversion strength analysis of uninjured and functionally unstable ankles. Journal of Athletic Training 1999; $34: 239-45$.

16 Franklin PJ, Kaminski TW, Horodyski MB, et al. An examination of eversion/inversion isokinetic strength ratios between uniniured and functionally unstable ankles. Journal of Athletic Training 1999;34:S72.

17 Baratta R, Solomonov M, Zhou BH, et al. Muscular coactivation: the role of the antagonist musculature in maintaining knee stability. Am J Sports Med 1988;16:113-22

18 Dranganich LF, Jaeger RJ, Krali AR. Coactivation of the hamstrings and quadriceps during extension of the knee. J Bone Joint Surg $[\mathrm{Am}]$ 1989;37:1075-81

19 Hole CD, Smith GH, Hammond J, et al. Dynamic control and conventional strength ratios of the quadriceps and hamstrings in subjects with anterior cruciate ligament deficiency. Ergonomics 2000;43: 1603-9.

20 Aagaard P, Simonsen EB, Magnusson SP, et al. A new concept for isokinetic hamstring: quadriceps muscle strength ratio. Am J Sports Med 1998;26:231-7.

21 Hartsell HD. Isokinetics and muscle strength ratios of the ankle invertors/evertors: a pilot study. Isokinetic Exercise Science 1994;4:116-21

22 Hartsell HD, Spaulding SJ. Eccentric/concentric ratios at selected velocities for the invertor and evertor muscles of the chronically unstable ankle. Br J Sports Med 1999;33:255-8.

23 Buckley BD, Kaminski TW, Powers ME, et al. Using reciprocal muscle group ratios to examine isokinetic strength in the ankle: a new concept. Journal of Athletic Training 2001;35:S93

24 Kaminski TW, Buckley BD, Powers ME, et al. Eversion and inversion strength ratios in subjects with unilateral functional instability. Med Sci Sports Exerc 2001;33:S135.

25 Dvir Z, Eger G, Halperin N, Shklar A. Thigh muscle activity and anterio cruciate ligament insufficiency. Clin Biomech 1989;4:87-91.

26 Hoffman M, Payne VG. The effects of proprioceptive ankle disk training on healthy subjects. J Orthop Sports Phys Ther 1995;21:90-3.

27 Gauffin H, Tropp H, Odenrick P. Effect of ankle disk training on postural control in patients with functional instability of the ankle joint. Int $J$ Sports Med 1988;9:141-4.

28 Uh BS, Beynnon BD, Helie BV, et al. The benefit of a single-leg strength training program for the muscles around the untrained ankle. Am J Sports Med 2000;28:568-73.

29 Docherty CL, Moore JH, Arnold BL. Effects of strength training on strength development and joint position sense in functionally unstable ankles. Journal of Athletic Training. 1998;33:310-14.

30 Blackburn T, Guskiewicz KM, Petschauer MA, et al. Balance and joint stability: the relative contributions of proprioception and muscular strength. Journal of Sport Rehabilitation 2000;9:315-28.

31 Tomaszewski D. "T-Band Kicks" ankle proprioception program. Athletic Trainer: Journal of the National Athletic Train Association $1991 ; 26 ; 216-217219,227$.

32 Highgenboten CL, Jackson AW, Meske NB. Concentric and eccentric torque comparisons for knee extension and flexion in young adult males and females using the Kinetic Communicator. Am J Sports Med 1988:16:234-7

33 Kaminski TW, Perrin DH, Mattacola CG, et al. The reliability and validity of ankle inversion and eversion torque measurements from the Kin Com II isokinetic dynamometer. Journal of Sport Rehabilitation 1995:4:210-18.

34 Barstow IK, Kaminski TW, Bishop MD. The effect of enhanced eccentric resistance training on forearm flexor strength: a randomized controlled trial. Med Sci Sports Exerc 1997;30:S206.

35 Seger JY, Arvidsson B, Thorstensson A. Specific effects of eccentric and concentric training on muscle strength and morphology in humans. Eur $J$ Appl Physiol 1998;79:49-57.

36 Baker AG, Webright WG, Perrin DH. Effect of a "T-band" kick training protocol on postural sway. Journal of Sport Rehabilitation 1998; 7: 122-7

37 Holme E, Magnusson SP, Becher K, et al. The effect of supervised rehabilitation on strength, postural sway, position sense and re-injury risk after acute ankle ligament sprain. Scand J Med Sci Sports 1999;9:104-9

38 Pintsaar A, Brynhildsen J, Tropp H. Postural corrections after standardised perturbations of single limb stance: effect of training and orthotic devices in patients with ankle instability. Br J Sports Med 1996;30: 151-5.
39 Matsusaka N, Yokoyama S, Tsurusaki T, et al. Effect of ankle disk training combined with tactile stimulation to the leg and foot on functional instability of the ankle. Am J Sports Med 2001;29:25-30.

40 Sheth $\mathbf{P}, \mathrm{Yu}$ B, Laskowski ER, et al. Ankle disk training influences reaction times of selected muscles in a simulated ankle sprain. $\mathrm{Am} J$ Sports Med 1997:25:538-43

41 Patterson RM, Stegink Jansen CW, Hogan HA, et al. Material properties of Thera-Band Tubing. Phys Ther 2001;81:1437-45.

42 Page $\mathbf{P}$, Labbe A. Predicted versus actual torque production of elastic and pulley resistance. Phys Ther 2000;80:S34.

\section{COMMENTARY}

In a well designed and thorough investigation, the authors examined the effect of a common clinical rehabilitation protocol for subjects with functional ankle instability. Their paper is important for many reasons. Functional ankle instability is a multifactorial entity that seems to involve mechanical, muscular, and neural factors. The determination of which of these factors, or what combinations thereof, is most important for identification and prevention remains elusive. This paper reports that a combination of strength, proprioception and strength, and proprioception exercises did not result in changes in the eversion to inversion isokinetic strength ratios when compared with controls. The authors correctly state that mode specificity and rigorousness of training may explain their lack of difference in strength gain. This is an important point; the lack of differences does not indicate a methodological flaw but a flaw in accepted clinical practice. The use of tubing exercises, although appropriate for early stage rehabilitation, are often performed at submaximal loads; although neural gains can be expected, especially in the early stages of rehabilitation, strength gains are probably not likely. This study shows that, for strength gains to be appreciated, resistance must be sufficiently challenging. McDonagh and Davies ${ }^{1}$ reported that loads less than $66 \%$ of maximum do not produce improvements in strength even with repetitions above 100 per session in untrained subjects. Therefore, if tubing is to be used for strengthening, the resistance should be individually referenced to an individual maximum. The starting point and progression of a rehabilitation programme should be individualised. We recently completed a study measuring the effect of a strength and proprioception training programme on subjects with functional ankle instability (unpublished). Like the aforementioned study, all subjects began the rehabilitation progression at the same starting point. Although this made sense from a scientific viewpoint, the initial few weeks of strengthening were not challenging to the stronger subjects. Therefore, the changes in strength were only apparent for subjects who trained at levels of about $70 \%$ of maximum. The take home point is that many rehabilitation programmes that use tubing exercises, although better than nothing, may be doing little to promote strength gains. Equally exciting in this paper is the use of eversion to inversion strength ratios. The authors have identified the muscle action that is necessary for dynamic strength control. Further study of the importance or manipulation of muscle group ratios may provide better understanding and ammunition in the treatment of functional ankle instability.

\section{G Mattacola}

Division of Athletic Training, College of Health Sciences, University of Kentucky, Lexington, KY 40536-0200, USA carlmat@uky.edu

\section{REFERENCE}

1 McDonagh M Davies C. Adaptive responses of mammalian skeletal muscle to exercise with high loads. Eur J Appl Physiol 1984;52:139-55. 